\title{
The Association between Symptoms of Original Autonomic Neuropathy and the Heart Rate Variability in Diabetics Article
}

\author{
Min-Young Chun, Hoon-Ki Park ${ }^{1, *}$, Hwan-Sik Hwang ${ }^{1}$, Jae-Il Han¹, Young-Joon \\ Chee ${ }^{2}$, Jong-Sill Lee ${ }^{3}$
}

Department of Global Medical Science, Sungshin Women's University College of Nursing, Seoul, ${ }^{1}$ Department of Family Medicine, Hanyang University College of Medicine, Seoul, ${ }^{2}$ Division of Electrical Engineering, Ulsan University College of Engineering, Ulsan, ${ }^{3}$ Department of Biomedical Engineering, Hanyang University, Seoul, Korea

Background: There are few tools to detect the diabetic autonomic neuropathy at an earlier stage. This study was conducted to investigate the association between symptoms of autonomic neuropathy and the heart rate variability (HRV) in diabetics.

Methods: Study subjects consisted of 50 diabetic patients and 30 outpatient hospital control patients at a university family medicine department. The patients completed a Korean version of composite autonomic symptom scale (COMPASS). Electrocardiography was recorded in the supine position, on standing, and during deep breathing, for 5 minutes each. HRV of frequency domain was calculated by power spectral analysis.

Results: The COMPASS score was higher in female diabetic patients compared with that in controls. Among 50 diabetic patients, the total COMPASS score correlated positively with normalized low frequency (LF) score (normalized units, n.u.) $(\mathrm{r}=0.62, \mathrm{P}<0.001)$ and low frequency/high frequency $(\mathrm{LF} / \mathrm{HF})(\mathrm{r}=0.77, \mathrm{P}<0.001)$, negatively with normalized HF score (n.u.) ( $\mathrm{r}=-0.59, \mathrm{P}<0.001)$ and RMSSD (square root of the mean of the sum of the square of differences between adjacent $\mathrm{NN}$ interval; $\mathrm{r}=-0.33, \mathrm{P}=0.031$ ). The decrease in $\mathrm{LF}$ (n.u) and the increase in HF (n.u) by deep breathing from the supine position were higher in diabetic patients compared with those in controls. The increase in LF (n.u) and the decrease in HF (n.u) by standing from the supine position were lower in diabetic patients compared with those in controls.

Conclusion: The COMPASS score correlated with some component score of the HRV in diabetics. The HRV may be used as a tool to detect diabetic autonomic neuropathy by augmentation with position change.

Keywords: Diabetic Autonomic Neuropathy; Heart Rate Variability; Composite Autonomic Symptom Scale

Received: September 20, 2008, Accepted: July 12, 2011

${ }^{*}$ Corresponding Author: Hoon-Ki Park

Tel: 82-2-2290-8740, Fax: 82-2-2281-7279

E-mail: hoonkp@hanyang.ac.kr

Korean Journal of Family Medicine

Copyright (C) 2011 The Korean Academy of Family Medicine

(c) This is an open-access article distributed under the terms of the Creative Commons Attribution Non-Commercial License (http://creativecommons.org/licenses/by-nc/3.0) which permits unrestricted noncommercial use, distribution, and reproduction in any medium, provided the original work is properly cited.

\section{INTRODUCTION}

Diabetic autonomic neuropathy may occur at any stage of diabetes, but it usually develops in patients who have had diabetes for 20 years or more. ${ }^{1)}$ Cardiovascular autonomic neuropathy may result in orthostatic hypotension, persistent sinus tachycardia, and asymptomatic myocardial infarction ${ }^{2)}$ which may predispose sudden death. ${ }^{3-5)}$

Therefore careful history taking and a thorough physical examination are important to screen the presence of diabetic 
neuropathy at an early stage of diabetes. Diagnosis of diabetic autonomic neuropathy primarily depends on several autonomic symptoms, but few studies validated symptom profile as a diagnostic tool. ${ }^{6,7)}$

The heart rate variability (HRV) is a physiological phenomenon which reflects the variety of time intervals between heart beats. It is measured by the variation in the beat-to-beat interval. The main inputs to make a HRV are the balance between the sympathetic nervous system (SNS) and the parasympathetic nervous system (PNS). By detecting HRV, we can compare the relative activity of the SNS with the PNS. The HRV is also used to evaluate the cardiovascular autonomic regulation. ${ }^{8)}$

Power spectral analysis demonstrates the HRV as a time domain and a frequency domain. As a time domain, RMSSD (square root of the mean of the sum of the square of differences between adjacent NN interval) reflects the parasympathetic activity. As a frequency domain, high frequency (HF) activity ( 0.4 to $0.15 \mathrm{~Hz}$ ), especially, has been linked to PNS activity. Less is known about the physiological inputs of the low frequency (LF) activity ( 0.04 to $0.15 \mathrm{~Hz}$ ), though recent consensus suggests it is influenced either by the SNS or a mixture of both the SNS and the PNS. The LF/HF ratio is known to be influenced by the sympathovagal balance., ${ }^{90}$

The composite autonomic symptom scale (COMPASS) is a self-completed questionnaire that is composed of 73 items. These questions are important and critical in the diagnosis of autonomic neuropathy. ${ }^{11)}$ The COMPASS has nine weighted subscale scores and total score is calculated by summing the individual subscale score. It has been proved to correlate objective indexes of autonomic function and autonomic symptom severity. ${ }^{12)}$ The HRV as a tool to measure autonomic balance may be related with the COMPASS symptom profile, but there was no study about it. If we know the association between these two measurements, we can use them complementary to each other because one is symptom-based, and the other is autonomic sign-based.

This study was conducted to investigate the association between symptoms of autonomic neuropathy and the HRV in diabetics.

\section{METHODS}

\section{Subjects}

Study subjects consisted of 50 diabetic patients ( 34 male, 16 female) and 30 outpatient hospital control patients (11 male, 19 female) at a university family medicine department from March 2008 through August 2008. Age of the study subjects ranged from 42 to 74 years old. Diagnosis and classification of diabetes were based on guidelines of the Expert Committee Report of the American Diabetes Association. ${ }^{13)}$

We excluded those with known arrhythmia, unstable angina, myocardial infarction, or cerebrovascular disease, and those who were taking any autonomic drug such as $\beta$-blocker, $\beta$-agonist, calcium channel blocker, or angiotensin-converting enzyme inhibitors, etc. The written consent was obtained from each patient before enrollment in the study. This study was approved by the Institutional Review Board of Hanyang University Hospital, in Seoul, Korea. As baseline clinical characteristics, patient's age, sex, body mass index, smoking status, alcohol intake, exercise, and average consumption of coffee were assessed. Systolic and diastolic blood pressures were measured by a mercury sphygmomanometer. The serum levels of fasting blood glucose, total cholesterol, triglyceride, high density lipoprotein cholesterol, low density lipoprotein cholesterol were measured by standard methods.

\section{Measurement of Autonomic Symptoms and the HRV}

\section{1) COMPASS}

The COMPASS has weighted 9 subscale scores that assess severity of symptoms in the following domains: orthostatic intolerance ( 9 items; range, 0 to 40 ), secretomotor dysfunction (8 items; range, 0 to 20), male sexual dysfunction( 8 items; range, 0 to 30), bladder dysfunction ( 3 items; range, 0 to 20), gastrointestinal symptoms ( 14 items; range, 0 to 40 ), pupillomotor symptoms (7 items; range, 0 to 5), vasomotor symptoms (11 items; range, 0 to 10 ), reflex syncope ( 5 items; range, 0 to 20 ), and sleep dysfunction ( 8 items; range, 0 to 15 ). The range of item score is from the minimum score of two to the maximum score of seven in each item. The total scores are calculated by summation of the individual item scores and the maximum score may be up 
to 200 (in man) or 170 (in woman). All subjects completed the questionnaire within 5 minutes.

\section{2) HRV}

Study subjects got the blood test at fasting state for 8 hours, avoiding alcoholic drink, caffeine beverage, and excessive exercise for 24 hours before the test. The measurement was done from 9:00 $\mathrm{AM}$ to noon at room temperature of $22-24^{\circ} \mathrm{C}$ in a quiet and comfortable environment. Participants took a rest for three minutes or more in the supine position and were oriented on the method of the test. Electrocardiography was recorded in the supine position, on standing, and during deep breathing (inspiration for 3 seconds and expiration for 3 seconds), for 5 minute each. The HRV of frequency domain was calculated by power spectral analysis.

\section{3) HRV data analysis}

The MATLAB ver. 7.04 (Mathwork Inc., Natick, MA, USA) reorganized the electrocardiography of each participant, then we deleted the abnormal heart beat and any artifact by using hand operated manual. Data of electrocardiography were digitalized by Analog to Digital Converter (MP150, BIOPAC Systems Inc., Goleta, CA, USA) and analyzed by the program of HRV Analysis ver. 1.1 (Department of Applied Physics, University of Kuopio, Eastern Finland, Finland). We calculated mean RR, RMSSD as a time domain, LF (0.04-0.15 Hz), HF (0.15-0.4 Hz), LF/HF ratio as a frequency domain by power spectral analysis. The LF and HF power were transformed into normalized units (n.u.). Transforming data into normalized units also helped to accentuate sympathovagal balance. Normalized units were calculated as follows: $\mathrm{LF}$ (n.u.) = LF power / TP-VLF power $\times 100$; $\mathrm{HF}$ (n.u.) $=$ HF power $/ \mathrm{TP}-\mathrm{VLF}$ power $\times 100(\mathrm{TP}$, total power; VLF, very low frequency).

\section{Statistical Analysis}

All clinical data were summarized as median and interquartile range. Continuous clinical data were compared between diabetic patients and controls by using Mann-Whitney U test, Student's t-test. Partial correlation coefficient, Pearson $r$ was calculated to examine the correlation between the total COMPASS score and HRV domain scores after adjusting confounding variables. We used SPSS ver. 15.0 (SPSS Inc., Chicago, IL, USA). We

Table 1. Baseline demographic characteristics of study subjects.

\begin{tabular}{|c|c|c|c|c|}
\hline \multirow{2}{*}{ Characteristics } & \multicolumn{2}{|c|}{ Male } & \multicolumn{2}{|c|}{ Female } \\
\hline & Diabetics $(n=34)$ & Non-diabetics $(\mathrm{n}=11)$ & Diabetics $(n=16)$ & Non-diabetics $(n=19)$ \\
\hline Age (y) & $52(48-66)$ & $53(45-63)$ & $61(50-65)$ & $60(53-65)$ \\
\hline Duration (y) & $7.0(3-10)$ & NA & $6.5(3-10)$ & NA \\
\hline $\operatorname{BMI}\left(\mathrm{kg} / \mathrm{m}^{2}\right)$ & $25.5(25.0-26.8)$ & $25.3(23.0-26.2)$ & $26.0(24.5-28.1)$ & $25.5(24.9-26.1)$ \\
\hline Smoking (pack·y) & $0(0-20)$ & $20(0-30)$ & $0(0-0)$ & $0(0-0)$ \\
\hline Coffee (cups/d) & $2(1-3)$ & $2(0-4)$ & $1(0-2)$ & $0(0-2)$ \\
\hline Exercise (h/d) & $1(0-1)$ & $1(0-2)$ & $1(0-1)$ & $1(0-1)$ \\
\hline Systolic BP (mm Hg) & $126(112-140)$ & $125(110-140)$ & $128(114-142)$ & $120(120-140)$ \\
\hline Diastolic BP (mm Hg) & $80(78-82)$ & $80(78-80)$ & $80(78-82)$ & $80(80-80)$ \\
\hline Fasting blood sugar (mg/dL) & $126(106-145)^{*}$ & $103(97-110)$ & $124(113-135)^{*}$ & $98(95-102)$ \\
\hline HDL-C (mg/dL) & $41(35-47)$ & $50(41-57)$ & $49(44-54)$ & $48(45-55)$ \\
\hline LDL-C (mg/dL) & $100(82-115)$ & $110(100-149)$ & $104(91-125)$ & $111(104-124)$ \\
\hline Triglyceride (mg/dL) & $116(90-179)$ & $122(69-203)$ & $115(102-140)^{\dagger}$ & $95(80-120)$ \\
\hline
\end{tabular}

Values are presented as median (interquartile range, $25-75 \%$ ).

BMI: body mass index calculated as weight in kilograms divided by height in meter squared, BP: blood pressure, HDL-C: high density lipoprotein cholesterol, LDL-C: low density lipoprotein cholesterol, NA: not available.

${ }^{*} \mathrm{P}<0.001 .{ }^{\dagger} \mathrm{P}<0.05$ vs. non-diabetics by Mann-Whitney U-test. 
considered it statistically significant if $\mathrm{P}$-values were below 0.05 .

\section{RESULTS}

\section{General Characteristics of the Study Sub-} jects

Diabetic patients had higher fasting glucose $(\mathrm{P}<0.001)$ both in men and in women group, low serum triglyceride $(\mathrm{P}<0.05)$ in women when compared with those of controls. All other clinical characteristics were not significantly different between diabetics and control group (Table 1).

\section{The COMPASS Score}

As a severity index of autonomic dysfunction, the total COMPASS score was higher in diabetic patients compared with that in controls. However, only female patients had significantly higher total COMPASS scores compared with the male controls (diabetics, 9.0; controls, 4.5; $\mathrm{P}<0.05$ ) (Table 2). Although data are not shown, among nine subscales, the subscale score of sleep

Table 2. The comparison of COMPASS score and HRV domain scores between diabetics and non-diabetics.

\begin{tabular}{|c|c|c|c|c|}
\hline \multirow{2}{*}{ Variables } & \multicolumn{2}{|c|}{ Male } & \multicolumn{2}{|c|}{ Female } \\
\hline & Diabetics $(\mathrm{n}=34)$ & Non-diabetics $(\mathrm{n}=11)$ & Diabetics $(n=16)$ & Non-diabetics $(\mathrm{n}=19)$ \\
\hline Total COMPASS scores & $12.5(8.0-16.0)$ & $6.5(1.5-22.5)$ & $9.0(3-15)^{*}$ & $4.5(2-14)$ \\
\hline \multicolumn{5}{|l|}{ Supine } \\
\hline $\mathrm{LF}\left(\mathrm{ms}^{2}\right)$ & $71.0(28-155)$ & $200.0(21-304)$ & $52.5(16-117)$ & $56.0(46-107)$ \\
\hline LF (n.u.) & $71.9(52-83)$ & $61.1(39-88)$ & $55.6(37-81)$ & $60.3(28-72)$ \\
\hline $\mathrm{HF}\left(\mathrm{ms}^{2}\right)$ & $33.0(16-56)$ & $49.0(17-204)$ & $28.5(11-103)$ & $39.0(29-117)$ \\
\hline HF (n.u.) & $28.2(17-39)$ & $38.9(12-46)$ & $44.4(19-63)$ & $39.7(28-72)$ \\
\hline $\mathrm{LF} / \mathrm{HF}$ & $2.6(1.6-4.9)$ & $1.6(0.6-7.5)$ & $1.3(0.6-4.5)$ & $1.5(0.6-2.5)$ \\
\hline RMSSD (ms) & $19.5(14.0-28.9)$ & $23.7(16.2-48.3)$ & $16.7(10.6-35.7)$ & $24.7(17.8-29.4)$ \\
\hline \multicolumn{5}{|l|}{ Deep breathing } \\
\hline $\mathrm{LF}\left(\mathrm{ms}^{2}\right)$ & $36.0(20-72)$ & $51.0(23-203)$ & $25.5(12-55)$ & $46.0(28-104)$ \\
\hline LF (n.u.) & $29.9(22-40)$ & $21.1(16-56)$ & $23.9(16-38)$ & $27.6(17-35)$ \\
\hline $\mathrm{HF}\left(\mathrm{ms}^{2}\right)$ & $84.0(27-240)$ & $121.0(35-209)$ & $100.0(31-181)$ & $162.0(44-272)$ \\
\hline HF (n.u.) & $70.2(60-78)$ & $78.9(44-84)$ & $76.1(62-84)$ & $72.4(65-83)$ \\
\hline $\mathrm{LF} / \mathrm{HF}$ & $0.4(0.3-0.7)$ & $0.3(0.2-1.3)$ & $0.3(0.2-0.6)$ & $0.4(0.2-0.6)$ \\
\hline RMSSD (ms) & $19.4(15.4-31.9)$ & $25.3(16.4-41.9)$ & $19.8(10.4-31.6)$ & $25.1(17.1-40.0)$ \\
\hline \multicolumn{5}{|l|}{ Standing } \\
\hline $\mathrm{LF}\left(\mathrm{ms}^{2}\right)$ & $73.5(42-140)$ & $58.4(19-259)$ & $41.5(27-56)$ & $58.0(32-84)$ \\
\hline LF (n.u.) & $82.5(72-85)$ & $71.1(63-85)$ & $64.7(37-75)$ & $74.4(62-82)$ \\
\hline $\mathrm{HF}\left(\mathrm{ms}^{2}\right)$ & $16.5(13-30)$ & $23.7(9-72)$ & $23.5(7-81)$ & $26.0(9-50)$ \\
\hline HF (n.u.) & $17.6(15-28)$ & $28.9(15-37)$ & $35.6(25-63)$ & $25.6(18-38)$ \\
\hline $\mathrm{LF} / \mathrm{HF}$ & $4.7(2.7-6.3)$ & $4.5(1.6-5.9)$ & $1.9(0.5-3.2)$ & $3.1(1.5-4.5)$ \\
\hline RMSSD (ms) & $18.2(12.6-25.2)$ & $12.1(9.3-23.1)$ & $19.6(10.6-23.7)$ & $16.3(9.9-20.2)$ \\
\hline
\end{tabular}

Values are presented as median (interquartile range, 25-75\%o).

COMPASS: composite autonomic symptom scale, LF: low frequency power $(0.004-0.15 \mathrm{~Hz})$, n.u.: normalized units, HF: high frequency power $(0.15-0.40 \mathrm{~Hz}), \mathrm{LF} / \mathrm{HF}$ : the ratio of low to high frequency power, RMSSD: square root of the mean of the sum of the square of differences between adjacent $\mathrm{NN}$ interval.

*P-value $<0.05$ was obtained by Mann-Whitney U test vs. non-diabetics. 
dysfunction was significantly higher only in female diabetics compared with that of female control group (1.5 [range, 1 to 3] vs. 0 [range, 0 to 2]; $\mathrm{P}<0.05)$.

\section{HRV Domain Scores}

In order to examine the characteristics of HRV domains (LF $\left[\mathrm{ms}^{2}\right]$, LF [n.u.], HF [ms $\left.{ }^{2}\right], \mathrm{HF}$ [n.u.], LF/HF, RMSSD [ms]) of diabetic patients, HRV domain scores in diabetic patients were compared with those of controls. All HRV domain scores were not significantly different between these two groups in the supine position, on standing, and during deep breathing (Table 2).

\section{The Correlation between COMPASS Scores and Each of HRV Domain Scores \\ Partial correlation coefficient between total COMPASS score} and HRV domain score was calculated after adjusting for age, sex, fasting blood glucose, body mass index, systolic blood pressure, diastolic blood pressure, smoking, high-density lipoprotein cholesterol, low-density lipoprotein cholesterol, and triglyceride. Among 50 diabetic patients, the total COMPASS score correlated positively with normalized LF score (n.u.) $(\mathrm{r}=0.62, \mathrm{P}<0.001)$ and LF/HF $(r=0.77, \mathrm{P}<0.001)$, negatively with normalized HF score (n.u.) $(\mathrm{r}=-0.59, \mathrm{P}<0.001)$ and RMSSD $(\mathrm{r}=-0.33, \mathrm{P}=$ 0.031) (Table 3).

\section{Difference between HRV Domain Scores in Supine Position, in Supine Position with Deep Breathing, and on Standing \\ In order to compare HRV responses to deep breathing and} standing in two groups, we measured the difference between HRV domain scores in the supine position, in the supine position with deep breathing, and on standing (Table 4$)$. The decrease in LF (n.u) $(P=0.04)$ and the increase in HF (n.u) $(P=0.04)$ by deep breathing from the supine position were higher in diabetic patients compared with those in controls. The increase in LF (n.u) $(\mathrm{P}=0.04)$ and the decrease in HF (n.u) $(\mathrm{P}=0.03)$ by standing from the supine position were lower in diabetic patients compared with those in controls.

\section{DISCUSSION}

Diabetic autonomic symptoms and signs manifest themselves definitely in pupil, sweat gland, urinary system, gastrointestinal system, adrenal system, and cardiovascular system. Antagonism and balance between sympathetic nerve and parasympathetic nerve effect on these systems. ${ }^{14)}$ The COMPASS is designed to provide a severity index of autonomic symptoms and gives different weights according to clinical importance. It yields one total score reflecting overall severity of autonomic symptoms. ${ }^{15)}$ In this study, we found that autonomic symptoms among

Table 3. The correlation between the composite autonomic symptom scale (COMPASS) scores and each of heart rate variability domain scores at three positions in 50 diabetic patients.

\begin{tabular}{|c|c|c|c|c|c|c|}
\hline \multirow{2}{*}{ Variables } & \multicolumn{2}{|c|}{ Supine } & \multicolumn{2}{|c|}{ With breathing control } & \multicolumn{2}{|c|}{ Standing } \\
\hline & $\mathrm{r}^{*}$ & P-value & $\mathrm{r}^{*}$ & P-value & $\mathrm{r}^{*}$ & P-value \\
\hline $\mathrm{LF}$ & 0.125 & 0.429 & -0.023 & 0.887 & 0.102 & 0.519 \\
\hline HF & -0.333 & 0.031 & -0.077 & 0.628 & -0.382 & 0.013 \\
\hline LF (n.u.) & 0.621 & $<0.001$ & 0.296 & 0.057 & 0.515 & 0.001 \\
\hline HF (n.u.) & -0.589 & $<0.001$ & -0.278 & 0.075 & -0.518 & $<0.001$ \\
\hline $\mathrm{LF} / \mathrm{HF}$ & 0.768 & $<0.001$ & 0.434 & 0.004 & 0.578 & $<0.001$ \\
\hline RMSSD (ms) & -0.334 & 0.031 & -0.175 & 0.269 & -0.274 & 0.079 \\
\hline
\end{tabular}

LF: low frequency, HF: high frequency, RMSSD: square root of the mean of the sum of the square of differences between adjacent NN interval.

*Partial correlation coefficient $r$ was adjusted by age, sex, fasting blood glucose, body mass index, systolic blood pressure, diastolic blood pressure, smoking, high density lipoprotein cholesterol, low density lipoprotein cholesterol, and triglyceride. 
Table 4. Difference between heart rate variability domain scores in supine position, in supine position with deep breathing, and on standing.

\begin{tabular}{|c|c|c|c|}
\hline Variables & $\begin{array}{l}\text { Diabetics } \\
(\mathrm{n}=50)\end{array}$ & $\begin{array}{l}\text { Non-diabetics } \\
\qquad(\mathrm{n}=30)\end{array}$ & P-value* \\
\hline \multicolumn{4}{|c|}{ Change from supine position to deep breathing position } \\
\hline$\Delta \mathrm{LF}\left(\mathrm{ms}^{2}\right)$ & $-47.1(105.9)$ & $-53.5(147.4)$ & 0.82 \\
\hline$\Delta \mathrm{LF}$ (n.u.) & $-33.4(21.3)$ & $-23.2(20.9)$ & 0.04 \\
\hline$\Delta \mathrm{HF}\left(\mathrm{ms}^{2}\right)$ & $104.6(239.8)$ & $135.7(353.4)$ & 0.64 \\
\hline$\Delta \mathrm{HF}(\mathrm{n} . \mathrm{u})$ & $33.4(21.4)$ & $23.5(21.2)$ & 0.04 \\
\hline$\Delta \mathrm{LF} / \mathrm{HF}$ & $-3.0(4.2)$ & $-1.8(2.6)$ & 0.16 \\
\hline$\triangle \mathrm{RMSSD}(\mathrm{ms})$ & $-1.3(18.2)$ & $-1.1(10.9)$ & 0.94 \\
\hline \multicolumn{4}{|c|}{ Change from supine position to standing position } \\
\hline$\Delta \mathrm{LF}\left(\mathrm{ms}^{2}\right)$ & $-12.9(88.8)$ & $-25.7(117.8)$ & 0.58 \\
\hline$\Delta \mathrm{LF}$ (n.u.) & $7.2(18.6)$ & $16.6(22.3)$ & 0.04 \\
\hline$\Delta \mathrm{HF}\left(\mathrm{ms}^{2}\right)$ & $-30.9(124.6)$ & $-70.3(216.8)$ & 0.30 \\
\hline$\Delta \mathrm{HF}$ (n.u) & $-6.5(17.8)$ & $-16.1(22.5)$ & 0.03 \\
\hline$\Delta \mathrm{LF} / \mathrm{HF}$ & $0.9(4.6)$ & $1.0(3.2)$ & 0.91 \\
\hline$\triangle \mathrm{RMSSD}(\mathrm{ms})$ & -19.3 (11.7) & $-4.9(21.7)$ & $<0.001$ \\
\hline
\end{tabular}

Values are presented as mean (SD).

LF: low frequency $(0.004-0.15 \mathrm{~Hz})$, n.u.: normalized units, HF: high frequency $(0.15-0.40 \mathrm{~Hz}), \mathrm{LF} / \mathrm{HF}$ : the ratio of low to high frequency power, RMSSD: square root of the mean of the sum of the square of differences between adjacent $\mathrm{NN}$ interval.

*P-value was obtained by Student's t-test.

the diabetic patients were significantly prevalent compared with control subjects. Among nine subscales, the orthostatic intolerance was the most frequent symptom. As the item score of orthostatic intolerance was highly weighted to make a total COMPASS score, it may be regarded as one of important determining factors for the total COMPASS score.

The COMPASS score correlated with some component scores of the HRV in diabetics. HRV domains related to SNS (normalized LF score and LF/HF ratio) correlated positively, but HRV domains related to PNS (normalized HF score, HF score, and RMSSD) did negatively with the total COMPASS score. Therefore, it suggests that the higher prevalence of autonomic symptoms in diabetic patients is, the higher activity of SNS in these patients is expected. Degeneration of the vagus nerve in an early stage ${ }^{9,16)}$ might result in relatively increased sympathetic activity at rest. It had been reported that high activity of SNS was the independent prognostic factor of hypertensive ventricular hypertrophy, morbidity and mortality of cardiovascular diseases. ${ }^{17-19)}$ Enabling risk stratification by screening for cardiac autonomic diabetic neuropathy (CADN) might facilitate early and successful intervention, even in relation to $\mathrm{CADN}^{20}{ }^{20}$

All of the HRV domain scores were not significantly different between the diabetic group and the control group. However, we found significant change of HRV response to deep breathing and standing in both groups. Generally, long expiration by deep breathing stimulates the PNS and results in increased HF score and decreased LF score. ${ }^{9,21)}$ On the other hand, standing stimulates SNS and results in decreased HF score and increased LF score. Especially, we found the change of HRV scores was lower in diabetic patients by augmentation with positional change. Therefore, it may be interpreted that the diabetic patients had lowered sympathetic responses to positional change like standing ${ }^{20,22)}$ and increased orthostatic hypotensive reaction. ${ }^{23)}$

There were several limitations in our study. Firstly, since a valid translation of a COMPASS questionnaire in Korean version had not been established, there might be a little bias due to cultural difference. Secondly, the questions about frequency and severity of autonomic symptoms were composed of somewhat vague items. However, we provided participants with additional explanation for the questions to overcome the subjectivity in rating the severity of symptoms. Because we didn't find any significant difference of the total COMPASS scores between men and women, we didn't further analyze correlation between COMPASS and HRV domain score in any one gender group.

In conclusion, the COMPASS score correlated with some component score of the HRVin diabetics. The HRV may be used as a tool to detect diabetic autonomic neuropathy by augmentation with position change.

\section{ACKNOWLEDGEMENTS}

This work was supported by a grant from Advanced Biometric Research Center (ABRC), Republic of Korea. 


\section{REFERENCES}

1. Aring AM, Jones DE, Falko JM. Evaluation and prevention of diabetic neuropathy. Am Fam Physician 2005;71:2123-8.

2. Ewing DJ, Campbell IW, Clarke BF. Assessment of cardiovascular effects in diabetic autonomic neuropathy and prognostic implications. Ann Intern Med 1980;92:308-11.

3. Campbell IW, Ewing DJ, Clarke BF. Painful myocardial infarction in severe diabetic autonomic neuropathy. Acta Diabetol Lat 1978;15:201-4.

4. Pickup JC, Williams G. Textbook of diabetes. Oxford: Blackwell Science; 1991.

5. Marchant B, Umachandran V, Stevenson R, Kopelman PG, Timmis AD. Silent myocardial ischemia: role of subclinical neuropathy in patients with and without diabetes. J Am Coll Cardiol 1993;22:1433-7.

6. Low PA, Vernino S, Suarez G. Autonomic dysfunction in peripheral nerve disease. Muscle Nerve 2003;27:646-61.

7. Joo NS, Park SB, Kim KM, Kim SM, Lee DJ, Kim BT. Changes in heart rate variability after weight reduction in the obese. J Korean Acad Fam Med 2005;26:693-8.

8. Choi WS, Ock SM, Kim CM, Lee BC, Jeong KS, Lee SJ. Effects of aerobic exercise on heart rate variability (HRV). J Korean Acad Fam Med 2005;26:561-6.

9. Eckberg DL. Sympathovagal balance: a critical appraisal. Circulation 1997;96:3224-32.

10. Low PA. Laboratory evaluation of autonomic function. In: Low PA, editor. Clinical autonomic disorders: evaluation and management. 2nd ed. Philadelphia: Lippincott-Raven; 1997. p.179-208.

11. Suarez GA, Opfer-Gehrking TL, Offord KP, Atkinson EJ, O'Brien PC, Low PA. The Autonomic Symptom Profile: a new instrument to assess autonomic symptoms. Neurology 1999;52:523-8.

12. Benrud-Larson LM, Dewar MS, Sandroni P, Rummans TA, Haythornthwaite JA, Low PA. Quality of life in patients with postural tachycardia syndrome. Mayo Clin Proc 2002;77:531-7.
13. Expert Committee on the Diagnosis and Classification of Diabetes Mellitus. Report of the expert committee on the diagnosis and classification of diabetes mellitus. Diabetes Care 2003;26 Suppl 1:S5-20.

14. Ziegler D, Gries FA, Spuler M, Lessmann F. The epidemiology of diabetic neuropathy. Diabetic Cardiovascular Autonomic Neuropathy Multicenter Study Group. J Diabetes Complications 1992;6:49-57.

15. Hosking DJ, Bennett T, Hampton JR. Diabetic autonomic neuropathy. Diabetes 1978;27:1043-55.

16. Ewing DJ, Campbell IW, Clarke BF. Mortality in diabetic autonomic neuropathy. Lancet 1976;1:601-3.

17. Ziegler D, Laux G, Dannehl K, Spuler M, Muhlen H, Mayer P, et al. Assessment of cardiovascular autonomic function: agerelated normal ranges and reproducibility of spectral analysis, vector analysis, and standard tests of heart rate variation and blood pressure responses. Diabet Med 1992;9:166-75.

18. Schlaich MP, Kaye DM, Lambert E, Sommerville M, Socratous F, Esler MD. Relation between cardiac sympathetic activity and hypertensive left ventricular hypertrophy. Circulation 2003;108:560-5.

19. Roy TM, Peterson HR, Snider HL, Cyrus J, Broadstone VL, Fell RD, et al. Autonomic influence on cardiovascular performance in diabetic subjects. Am J Med 1989;87:382-8.

20. Schonauer M, Thomas A, Morbach S, Niebauer J, Schonauer U, Thiele H. Cardiac autonomic diabetic neuropathy. Diab Vasc Dis Res 2008;5:336-44.

21. Low PA, Benrud-Larson LM, Sletten DM, Opfer-Gehrking TL, Weigand SD, O'Brien PC, et al. Autonomic symptoms and diabetic neuropathy: a population-based study. Diabetes Care 2004;27:2942-7.

22. Jacob G, Atkinson D, Jordan J, Shannon JR, Furlan R, Black $\mathrm{BK}$, et al. Effects of standing on cerebrovascular resistance in patients with idiopathic orthostatic intolerance. Am J Med 1999;106:59-64.

23. Hilsted J, Parving HH, Christensen NJ, Benn J, Galbo H. Hemodynamics in diabetic orthostatic hypotension. J Clin Invest 1981;68:1427-34 